\title{
Evaluation of subcutaneous rituximab administration on Canadian systemic therapy suites
}

\author{
D.A. Stewart MD, ${ }^{*}$ J.S. Boudreault $\mathrm{MD}_{1}^{\dagger}$ B. Maturi $\mathrm{MSc}_{,}^{\ddagger}$ D. Boras $\mathrm{MD}_{1}^{\ddagger}$ and R. Foley $\mathrm{MD}^{\S}$
}

\begin{abstract}
Background Non-Hodgkin lymphoma (NHL) is the most common hematologic malignancy. Diffuse large B-cell lymphoma (DLBCL) and follicular lymphoma (FL) constitute 55\% of new NHL cases and are initially treated with rituximab-based chemoimmunotherapy. Relative to intravenous (IV) rituximab, a subcutaneous (sc) formulation approved in 2016 has comparable pharmacokinetics, efficacy, and safety, and a greatly reduced administration time; it is also preferred by patients. The objective of the present study was to estimate the effect (on systemic therapy suite time and on the costs of drug acquisition and administration) of implementing sc rituximab in the initial chemoimmunotherapy for FL and DLBCL over 3 years in the Canadian market.
\end{abstract}

Methods An Excel (Microsoft Corporation, Redmond, WA, U.S.A.)-based model was created with a population size based on epidemiologic data and current rituximab use, duration of use considering initial therapy, time savings for sc rituximab administration from published studies, costs from standard Canadian sources, and assumed uptake in implementing provinces of $65 \%, 75 \%$, and $80 \%$ over 3 years. Key parameters and sensitivity analysis values were validated by clinical experts located in various Canadian jurisdictions. Costs are reported in 2017 Canadian dollars from the perspective of the health care system.

Results More than 3 years after implementation of sc rituximab, we estimated that 5762 Canadians would be receiving sc rituximab, resulting in savings of 128,715 hours in systemic therapy suite time and approximately $\$ 40$ million in drug and administration costs. Sensitivity analyses suggest that the model is most sensitive to sc market uptake, number of induction therapy cycles, and eligible patients.

Conclusions Subcutaneous administration of rituximab can significantly reduce systemic therapy suite time and achieve substantial savings in drug and administration costs.

Key Words Non-Hodgkin lymphoma, rituximab, subcutaneous rituximab, intravenous rituximab, cost analyses

Curr Oncol. 2018 Oct;25(5):300-306 www.current-oncology.com

\section{INTRODUCTION}

Non-Hodgkin lymphoma (NHL) is the most common hematologic malignancy in Canadian adults ${ }^{1}$, with an estimated 8300 new cases diagnosed and 2700 attributable deaths in $2017^{2}$. The CD20-positive B-cell lymphomas constitute $85 \%-90 \%$ of NHL, with diffuse large B-cell lymphoma (DLBCL) and follicular lymphoma (FL) making up more than half of new NHL cases ${ }^{1}$.

In Canada, standard frontline treatment for DLBCL and FL involves rituximab-based chemoimmunotherapy, traditionally with an intravenous (IV) infusion of rituximab $\left(375 \mathrm{mg} / \mathrm{m}^{2}\right)^{3,4}$. In Canada, the standard treatment for DLBCL is rituximab-CHOP (cyclophosphamidedoxorubicin-vincristine-prednisone) for 6 cycles, without rituximab maintenance. For FL, patients receive 6 cycles of rituximab-bendamustine [or 6-8 cycles of rituximab-CVP (cyclophosphamide-vincristine-prednisone)], followed by 8 cycles of rituximab maintenance therapy given every 3 months, except in Quebec, where rituximab maintenance is not used after rituximab-bendamustine. Studies have demonstrated that patients receiving IV rituximab in 
addition to chemotherapy not only experience improved progression-free survival, but also superior overall surviv$\mathrm{al}^{5-7}$. The preparation and administration of IV rituximab is resource-intensive, requiring 2-6 hours to calculate the appropriate dose based on body surface area (BSA), to prepare fluids, and to titrate and administer the infusion $^{4,8-10}$ —all of which occur in addition to the preparation and administration time associated with chemotherapy treatment, which is administered sequentially.

Recently, a subcutaneous (sc) formulation of rituximab was approved by Health Canada for use in DLBCL and $\mathrm{FL}^{11}$. The sc formulation has the same active ingredient as IV rituximab, but it has been concentrated to one twelfth of the IV format, with the enzyme recombinant human hyaluronidase acting as a permeation enhancer, increasing dispersion and absorption to support administration of the rituximab in high volume ${ }^{4,8,12}$.

After one initial IV administration of rituximab is tolerated, sc rituximab administered as a fixed dose provides an alternative to the IV formulation in DLBCL and FL, as currently used in rituximab-containing regimens. The sc administration of rituximab takes significantly less time than IV administration ${ }^{9,13}$; however, to monitor for side effects, administration must still occur in a systemic therapy suite ${ }^{11}$. Nevertheless, sc rituximab simplifies administration and reduces the treatment burden both for patients and for already constrained health care resources, including systemic therapy suite time.

Phase I-III studies comparing sc with IV rituximab for FL and DLBCL have demonstrated similar pharmacokinetics, similarity in rates of overall response and complete response $(\mathrm{CR})$ or unconfirmed $\mathrm{CR}$, and similar safety profiles (with the exception of an increase in mild, but expected, local injection site reactions for sc rituximab) ${ }^{9,13,14}$.

Large proportions of nurses $(94.6 \%)$ and patients (92.9\%) prefer sc administration of rituximab, with reasons for that preference being reduced clinic time, increased comfort during administration, and less emotional distress ${ }^{15}$.

A decision to administer sc rituximab therefore has the potential to reduce the overall burden on systemic therapy suites, making that valuable and constrained health care system resource available for the treatment of other patients while maintaining the safety and effectiveness of rituximab treatment and improving patient satisfaction ${ }^{8}$. Making this change in administration format, with its reduced use of systemic therapy suites, is also expected to lower the cost to the health care system.

To date, several provinces in Canada have begun using the sc formulation; others are considering its use. In Quebec, the sc formulation is not being implemented at this time ${ }^{14}$, and IV administration of rituximab for all patients is assumed for Quebec in the present analysis.

Given the potential resource savings associated with the implementation of sc rituximab, we aimed to

estimate, on a population level, savings in the form of systemic therapy suite time that would be freed with the implementation of sc rituximab in the Canadian market; and

- estimate the aggregate cost impact of implementing sc rituximab in the Canadian market, including the acquisition cost for rituximab and administration costs associated with each therapy.

The findings from this study will support clinicians and health care administrators as they consider whether to implement sc rituximab into their clinical processes.

\section{METHODS}

Using a health care system perspective, the impact on systemic therapy suite time, rituximab acquisition, and administration costs of providing sc rituximab were estimated. An Excel (Microsoft Corporation, Redmond, WA, U.S.A.)-based model was used to estimate annual systemic therapy suite time saved and the annual net cost impact of drug acquisition and administration, including hospital and physician services. Year 1 of the 3 -year analysis was assumed to begin January 2018. Key input parameters of the model were based on literature and chart review data and were validated by the clinical authors of this paper (DAS, RF, JSB) and by therapeutic-area experts (TAEs) located in three distinct jurisdictions of Canada (Ontario, Alberta, and Quebec).

The chart review included 762 charts collected from several provinces across Canada, including Ontario, Quebec, British Columbia, and several of the Atlantic provinces. National estimates were determined by weighting the data collected from the various provinces to reflect their expected proportional contribution according to Canadian Cancer Statistics $2017^{1}$. The expert clinicians, working primarily in oncology settings, validated the appropriateness of the chart review and best-available-literature estimates to reflect Canadian practice. Sensitivity analyses (sAs) for key parameters were conducted to reflect variations in province-specific estimates. Costs are reported in 2017 Canadian dollars.

\section{Key Parameters}

\section{Population and Market Assumptions}

The target population (incident patients with DLBCL or FL), corresponding to the clinical indication for rituximab in Canada $^{11}$, was estimated using an epidemiologic approach, assuming that the sc formulation would be adopted where IV rituximab is currently used. A Canadian-specific NHL incidence rate from the Canadian Cancer Society ${ }^{1}$ of 20.8 cases per 100,000 individuals was applied to expected population estimates for 2015-2017 from Statistics Canada ${ }^{16}$ and projected to 2018-2020, using an annual growth rate of $1 \%$. Expected NHL incidence was further stratified by the proportions of DLBCL (33\%) and FL patients (22\%), as reported by the Canadian Cancer Society ${ }^{1}$.

It was estimated that, because of being elderly and frail, being diagnosed at a later stage, or feeling that they cannot tolerate treatment, $10 \%$ of individuals diagnosed with DLBCL and $15 \%$ of those diagnosed with FL might not proceed to discuss treatment options with their hematologist or medical oncologist. For patients presenting to the hematologist or medical oncologist to discuss treatment, the base-case analysis assumes that $96 \%$ of those with DLBCL and $90 \%$ of those with FL will receive rituximab. For DLBCL, rituximab 
is the primary frontline treatment choice, and for FL, $90 \%$ of patients overall receive rituximab, some as immediate treatment, and some after an initial watch-and-wait strategy — that is, they are assumed to have been diagnosed in a prior year and are now receiving rituximab in the current year (estimated by chart review of treatment in patients with FL). Table I summarizes the eligible Canadianwide population of DLBCL and FL patients receiving rituximab for years 2018-2020. The sA values varied by $\pm 20 \%$ from the base-case estimates (Table II).

\section{Scenarios}

The impact on systemic therapy suite time was estimated by comparing two scenarios. The first represented a world without availability of sc rituximab, in which only IV rituximab was available for use in Canada. In an alternative scenario, sc rituximab was available for use and was

TABLE I Population eligible for rituximab treatment ${ }^{\mathrm{a}}$

\begin{tabular}{lccc}
\hline \multicolumn{1}{c}{ Parameter } & \multicolumn{3}{c}{ Study year } \\
\cline { 2 - 4 } & $\mathbf{1}$ & $\mathbf{2}$ & $\mathbf{3}$ \\
\hline Incident patients $^{\mathrm{b}}$ in Canada & 4242 & 4284 & 4327 \\
Patients seeking treatment $^{\mathrm{c}}$ & 3733 & 3770 & 3808 \\
\hline Eligible cases treated with rituximab & 3359 & 3393 & 3427
\end{tabular}

a Includes patients with diffuse large B-cell lymphoma (DLBCL) and follicular lymphoma (FL).

b Calculated as the Canadian-based non-Hodgkin lymphoma (NHL) incidence rate multiplied by the total Canadian population estimates for 2018-2020, considering that DLBCL and FL respectively account for $33 \%$ and $22 \%$ of all patients with NHL.

c Further stratifies incident patients with DLBCL and FL by the proportions assumed to undertake treatment ( $90 \%$ for DLBCL and $85 \%$ for $\mathrm{FL}$ ). assumed to be used in varying market-share proportions of the initial induction IV rituximab use each year.

\section{Market Uptake}

The base-case analysis assumed sc formulation uptake of $65 \%, 75 \%$, and $80 \%$ of the IV rituximab market in years 1,2 , and 3 respectively for provinces implementing sc rituximab. Because of Quebec nonparticipation, the Canada-wide estimates were adjusted to $50 \%, 58 \%$, and $62 \%$ in years 1,2 , and 3 respectively. Table III summarizes the distribution of patients by scenario and market share. The lower sa value was $50 \%$ for all 3 years, and the upper sa values were $90 \%$, $95 \%$, and $100 \%$ for years 1,2 , and 3 respectively.

\section{Therapy Considerations}

Patients receiving IV rituximab as single-agent induction were not included, because sc rituximab is not approved for monotherapy induction. Furthermore, DLBCL patients received sc rituximab during induction only; FL patients received sc rituximab during the induction and maintenance phases. Based on chart review data, $85 \%$ of FL patients who receive induction therapy are assumed to respond well and are eligible for maintenance therapy. For many provinces, sc rituximab would be considered a switch in administration route from the IV formulation and would therefore be used in all DLBCL and FL indications in which IV rituximab is currently used, including in combination with multiple chemotherapy agents or bendamustine, given that bendamustine-rituximab induction followed by rituximab maintenance is commonly used. The sA values varied by $\pm 20 \%$ from the base-case estimates.

Based on chart review and validation by TAEs, the base-case analysis assumed an average of 5.6 three-weekly cycles of induction therapy and 7.1 cycles of maintenance therapy given every 3 months (weighted average of

TABLE II Key model parameters and alternative values for the sensitivity analyses

\begin{tabular}{|c|c|c|c|c|c|c|c|c|c|}
\hline \multirow[t]{3}{*}{ Parameter } & \multicolumn{3}{|c|}{ Base case } & \multicolumn{6}{|c|}{ Sensitivity analyses } \\
\hline & \multirow[t]{2}{*}{ Year 1} & \multirow[t]{2}{*}{ Year 2} & \multirow[t]{2}{*}{ Year 3} & \multicolumn{3}{|c|}{ Lower } & \multicolumn{3}{|c|}{ Upper } \\
\hline & & & & Year 1 & Year 2 & Year 3 & Year 1 & Year 2 & Year 3 \\
\hline Eligible patients $(n)^{\mathrm{a}}$ & 3359 & 3393 & 3427 & 2687 & 2714 & 2742 & 4031 & 4072 & 4112 \\
\hline Market capture for SC rituximab (\%) & 50 & 58 & 62 & 50 & 50 & 50 & 90 & 95 & 100 \\
\hline Patients with drug wastage $(\%)$ & & 10 & & & 0 & & & 20 & \\
\hline \multicolumn{10}{|l|}{ Costs (CA\$, 2017) } \\
\hline IV $100 \mathrm{mg}$ format & & 471 & & & 453 & & & 489 & \\
\hline IV $500 \mathrm{mg}$ format & & 2353 & & & 2266 & & & 2447 & \\
\hline Systemic therapy suite hour & & 199 & & & 159 & & & 239 & \\
\hline \multicolumn{10}{|l|}{ Savings per administration } \\
\hline Systemic therapy suite time (minutes) & & 199 & & & 159 & & & 238 & \\
\hline \multicolumn{10}{|l|}{ Cycles (n) } \\
\hline Induction therapy & & 6 & & & 4 & & & 8 & \\
\hline Maintenance therapy & & 7 & & & 4 & & & 8 & \\
\hline Patients with FL receiving maintenance (\%) & & 75 & & & 60 & & & 90 & \\
\hline
\end{tabular}

a Includes patients with diffuse large B-cell lymphoma (DLBCL) and follicular lymphoma (FL). 
TABLE III Patients receiving intravenous (IV) or subcutaneous (SC) rituximab with and without implementation of the SC formulation ${ }^{a}$

\begin{tabular}{|c|c|c|c|}
\hline \multirow{2}{*}{$\begin{array}{l}\text { Drug } \\
\text { formulation } \\
\text { availability }\end{array}$} & \multicolumn{3}{|c|}{$\begin{array}{l}\text { Patients receiving the formulation } \\
\text { in study year ... }\end{array}$} \\
\hline & 1 & 2 & 3 \\
\hline \multicolumn{4}{|l|}{ Only IV } \\
\hline IV & 3359 & 3393 & 3427 \\
\hline \multicolumn{4}{|l|}{ Both SC and IV } \\
\hline IV & 1675 & 1430 & 1312 \\
\hline SC & 1684 & 1963 & 2115 \\
\hline
\end{tabular}

first-line and later-line use). During the induction phase, patients were assumed to receive the IV formulation for their first dose before starting the sc formulation. The sA values ranged between 4 and 8 cycles of IV therapy during induction and maintenance.

The average BSA of patients with DLBCL and FL was based on the Canadian chart review and provincial data. Average estimates were derived based on the distribution of patients within the BSA ranges. The midpoint of each BSA range was used in the acquisition cost calculation for IV rituximab. Provincial estimates, when available, were used directly; otherwise, national average BSAs $\left(1.868 \mathrm{~m}^{2}\right.$ for DLBCL and $1.844 \mathrm{~m}^{2}$ for FL) were used (DeltaPA Database: IQVIA, Kirkland, QC).

Drug wastage for IV rituximab was considered and assumed to be minimal in real-world settings, given that several large centres across Canada work to minimize wastage by introducing strategies such as "rituximab days," during which patients who require rituximab are scheduled for therapy on the same day or days, such that centres can combine fractional drug leftovers for subsequent patients. Another commonly used strategy is "dose banding," in which patients are categorized and receive standard dosing rather than dosing customized to BSA. The base case therefore reflected use of those methods, assuming that drug wastage would be generated by only $10 \%$ of patients; the sA values varied by $\pm 10 \%$.

\section{Systemic Therapy Suite Time Considerations}

A time-and-motion study conducted alongside a phase III randomized controlled trial in the United Kingdom observed the time required for health care professionals to prepare and administer IV and sc rituximab, and also the time spent by the patient in the treatment room and the infusion chair ${ }^{9}$. The total systemic therapy suite time was estimated as the patient bed or chair time plus the drug preparation task time and was validated by TAEs as being aligned with their expectations. The total systemic therapy suite time for IV rituximab was reported to be 278 minutes per therapy session, compared with 79 minutes per sc rituximab therapy session ${ }^{9}$. The net time savings in systemic therapy suite time between sc and IV rituximab was therefore 199 minutes per therapy session. The sA varied the time savings by $\pm 20 \%$.

\section{Costs}

The costs of companion chemotherapy, adverse events management, and downstream health care were excluded from the analysis, given that they were expected to be equal for each scenario. The base-case list price for IV rituximab (\$470.515 per $100 \mathrm{mg}$ vial, $\$ 2352.590$ per $500 \mathrm{mg}$ vial) was sourced from the Ontario Exceptional Access Program, and the price for sc rituximab ( $\$ 2,888.51$ per $1400 \mathrm{mg}$ vial) is listed publicly in Quebec and reflects an acquisition cost per rituximab administration slightly lower than that for IV rituximab [AQPP (Association québécoise des pharmaciens propriétaires) Listed Price (DeltaPA Database: IQVIA, Kirkland, QC)]. The lower sa value for IV rituximab reflected the lowest public acquisition price in Canada, available from the Régie de l'assurance maladie Québec, and the upper sA value reflected a $4 \%$ increase from the base case.

The aggregate systemic therapy suite per-hour cost of \$178 (2010 Canadian dollars), including drug preparation, chair time, pharmacist and nurse wages, and overhead, was obtained from an Ontario-based study examining the cost effectiveness of systemic therapies for pancreatic cancer ${ }^{17}$. The systemic therapy suite time cost was calculated by multiplying the time impacts of IV and sc rituximab from the U.K. time-and-motion study ${ }^{9}$ by the per-hour systemic therapy suite cost, and then adjusting the result to 2017 Canadian dollars (\$199.05) based on the Bank of Canada consumer price index. The sA values varied by $\pm 20 \%$ from the base-case estimates. Physician fees for chemotherapy administration and oncologist visits were added based on the Ontario Health Insurance Plan Schedule of Benefits for Physician Services ${ }^{18}$.

Table II shows the key base-case parameter assumptions and the upper and lower sa values.

\section{RESULTS}

During the 3 years after implementation of sc rituximab, 1684,1963 , and 2115 patients were estimated to receive that formulation in years 1, 2, and 3 respectively (Table III), leading to an incremental savings in systemic therapy suite time of 32,063 hours in year 1, 45,995 hours in year 2 , and 50,656 hours in year 3 , and an incremental cost savings of approximately $\$ 10$ million, $\$ 14$ million, and $\$ 16$ million respectively (Table IV). Overall, implementation translates into 5762 patients using the sc formulation, with associated overall savings of 128,715 hours of systemic therapy suite time and approximately $\$ 40$ million dollars in rituximab acquisition and administration costs. Incremental time and administration cost savings are greater for patients with FL than for those with DLBCL, with $56 \%-58 \%$ of the time and cost savings being accrued by the FL patients. The incremental savings for 1 full course of treatment are $\$ 5,017$ (cost) and 15.28 systemic therapy suite hours per DLBCL patient, and \$12,212 and 38.8 hours per FL patient.

The sas showed that our model is most sensitive to the market uptake of sc rituximab over 3 years, with savings in systemic therapy suite time ranging from 114,530 to 216,778 hours and associated drug and administration cost savings ranging from approximately $\$ 36$ million to $\$ 68$ million. The next most influential parameter was the number of cycles of induction therapy, with systemic suite therapy 
TABLE IV Impact of subcutaneous (SC) rituximab on systemic therapy suite time and administration cost

\begin{tabular}{|c|c|c|c|c|}
\hline \multirow[t]{2}{*}{ Variable } & \multicolumn{4}{|c|}{ Study year } \\
\hline & 1 & 2 & 3 & Overall \\
\hline \multicolumn{5}{|l|}{ Systemic therapy suite time (hours) } \\
\hline Current scenario: rituximab IV only & 104,925 & 130,028 & 132,291 & 367,244 \\
\hline SC implementation scenario & 72,861 & 84,033 & 81,634 & 238,529 \\
\hline \multicolumn{5}{|l|}{ Incremental savings (CA\$, 2017) } \\
\hline Total & 32,063 & 45,955 & 50,656 & 128,715 \\
\hline Patients with FL & 16,278 & 27,595 & 30,832 & 74,705 \\
\hline Patients with DLBCL & 15,785 & 18,400 & 19,824 & 54,009 \\
\hline \multicolumn{5}{|l|}{ Administration cost (CA\$, 2017) } \\
\hline Current scenario: rituximab IV only & $23,448,090$ & $29,058,145$ & $29,563,785$ & $82,070,019$ \\
\hline SC implementation scenario & $17,065,918$ & $19,902,854$ & $19,480,616$ & $56,449,388$ \\
\hline \multicolumn{5}{|l|}{ Incremental savings ${ }^{\mathrm{a}}(\mathrm{CA} \$, 2017)$} \\
\hline Total & $6,382,172$ & $9,155,290$ & $10,083,169$ & $25,620,632$ \\
\hline Patients with FL & $3,240,070$ & $5,492,846$ & $6,137,158$ & $14,870,074$ \\
\hline Patients with DLBCL & $3,142,103$ & $3,662,444$ & $3,946,011$ & $10,750,558$ \\
\hline \multicolumn{5}{|c|}{ Total drug and administration cost (CA\$, 2017) } \\
\hline Current scenario: rituximab IV only & $95,000,136$ & $114,028,505$ & $115,892,336$ & $324,920,977$ \\
\hline SC implementation scenario & $84,869,229$ & $99,756,912$ & $100,201,482$ & $284,827,623$ \\
\hline \multicolumn{5}{|l|}{ Incremental savings ${ }^{\mathrm{a}}(\mathrm{CA} \$, 2017)$} \\
\hline Total & $10,130,907$ & $14,271,593$ & $15,690,854$ & $40,093,353$ \\
\hline Patients with FL & $4,942,095$ & $8,223,499$ & $9,174,483$ & $22,340,077$ \\
\hline Patients with DLBCL & $5,188,811$ & $6,048,094$ & $6,516,371$ & $17,753,276$ \\
\hline
\end{tabular}

a Calculated as the difference between the current scenario and the SC implementation scenario.

IV = intravenous; FL = follicular lymphoma; $\mathrm{DLBCL}=$ diffuse large B-cell lymphoma.

time saved ranging from 99,345 to 171,393 hours, and the associated cost ranging from approximately $\$ 31$ million to \$54 million (Figures 1 and 2).

\section{DISCUSSION}

Several studies support the use of sc rituximab in preference to IV rituximab for NHL. In patients with FL, the pharmacokinetics of fixed-dose sc rituximab (1400 mg) compared with standard IV rituximab $\left(375 \mathrm{mg} / \mathrm{m}^{2}\right)$ demonstrate noninferiority in the trough and area-underthe-curve concentrations ${ }^{8,12}$. Efficacy studies have shown treatment response to be similar for the sc and IV formulations. In treatment-naïve patients with FL, induction using sc rituximab with cHOP or cVP chemotherapy and subsequent maintenance showed similar overall response rates [IV rituximab: $84.9 \%$; 95\% confidence interval (CI): $79.2 \%$ to $89.5 \%$; sc rituximab: $84.4 \%$; $95 \%$ CI: $78.8 \%$ to $89.1 \%$ ] and equal CR point estimates $(32.2 \% \text {; } 95 \% \text { CI: } 25.9 \% \text { to } 39.1 \%)^{9,13}$. In treatment-naïve patients with DLBCL, induction using SC rituximab with CHOP showed similar overall response rates (IV rituximab: $78.0 \%$; 95\% cI: $71.1 \%$ to $83.8 \%$; sc rituximab: $82.2 \%$; $95 \%$ CI: $77.7 \%$ to $86.1 \%$ ) and CR rates that were not significantly different (IV rituximab: $42.4 \%$; 95\% cI: $35.1 \%$ to $49.7 \%$; sc rituximab: $50.6 \%$; $95 \%$ CI: $45.3 \%$ to $55.9 \%$; $p=0.076)^{19}$. In all the studies, safety profiles were similar, with

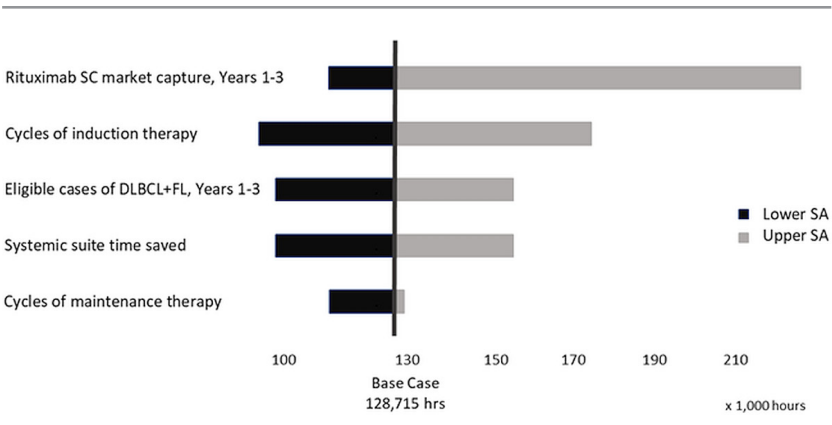

FIGURE 1 Tornado diagram for total savings in systemic therapy suite time. SC = subcutaneous; DLBCL = diffuse large B-cell lymphoma; FL = follicular lymphoma; $\mathrm{SA}=$ sensitivity analysis.

the exception of an expected increase in administrationrelated reactions for rituximab sc that were generally mild and transient ${ }^{4,8,12,19}$.

Additionally, compared with the IV formulation, the sc formulation was shown to provide significant time savings ${ }^{8,9,12,15,19}$, with a median administration time of 6 minutes $^{8,12}$ and relative reductions in active health care provider time of $27 \%-58 \%$ and patient chair time of $53 \%-91 \%^{13}$. 


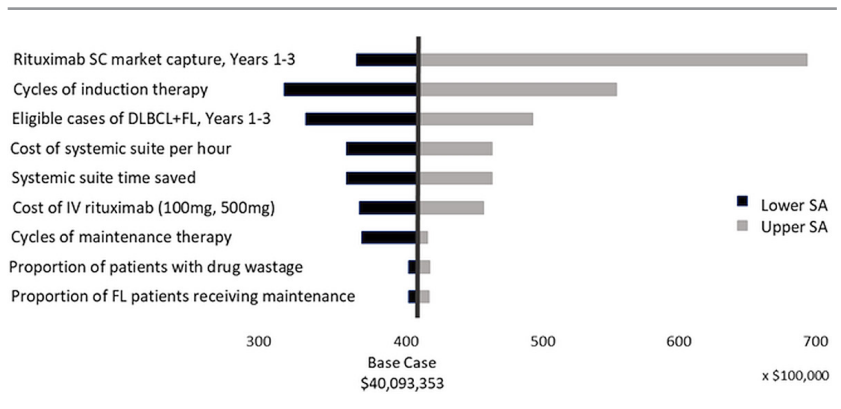

FIGURE 2 Tornado diagram for total rituximab acquisition and administration cost. $\mathrm{SC}=$ subcutaneous; $\mathrm{DLBCL}=$ diffuse large B-cell lymphoma; $\mathrm{FL}=$ follicular lymphoma; IV = intravenous; $\mathrm{SA}$ = sensitivity analysis.

Implementing sc rituximab as an alternative to IV rituximab for DLBCL and FL patients would lead to its use for an estimated 5762 patients and would increase the systemic therapy suite time available for other patients by a total of 128,715 hours over 3 years, assuming that the sc formulation is administered within a monitored systemic therapy suite setting and that, by the 3rd year of adoption, sc reaches $80 \%$ market uptake in provinces other than Quebec. Given that systemic therapy suites are a valuable but constrained resource in Canada, treatments that reduce the burden of suite-time use should be considered a priority for implementation. Use of the sc formulation is further associated with savings of approximately $\$ 26$ million in administrative health care resources and approximately $\$ 40$ million if the rituximab acquisition costs over 3 years are included.

The greatest impact on savings in systemic therapy suite time is related to the proportion of the population that will be given the sc formulation in preference to the IV formulation, and yet institutions and clinicians in provinces across Canada that are implementing the sc formulation might differ in whether sc is used as the institutional default or whether use of the sc formulation is a clinician decision. At the time of our analysis, the sc formulation was not funded in Quebec, but Alberta had completely defaulted to sc rituximab for all NHL patients and used the IV formulation only for those rare patients who can't tolerate the sc formulation. Other provinces have also seen varying levels of adoption. We did not evaluate the potential additional time and cost savings associated with the use of SC rituximab for other CD20-positive NHL subtypes, although similar savings could be expected when rituximab becomes available for chronic lymphocytic leukemia.

Introduction of other new treatments might also affect the use of rituximab overall. If a $20 \%$ reduction in population is assumed, 102,960 hours of systemic therapy suite time are still saved, together with an approximately \$32 million of cost savings for rituximab acquisition and administration. An IV rituximab biosimilar could soon become available, and at that time, an adjustment to the sc formulation's pricing would be required to maintain budget neutrality at the system level.

Drug wastage is a concern for costly medications, and the fixed dose for the sc formulation of rituximab avoids that problem, increasing its cost advantage given the greater wastage with the IV formulation. The base-case analysis conservatively assumed drug wastage for only $10 \%$ of patients receiving IV rituximab, given that most centres use the fractional leftover amounts of rituximab to treat subsequent patients during the same day and also use dose-banding calculations to minimize wastage.

Limitations to this study do exist. Our analysis estimates the impact on health care costs across Canada, but uses unit costs from a single province as a proxy for national costs. This accepted method for Canadian economic analyses is used, given that providing 10 provincial analyses is beyond the scope of the present work. The estimated patient population might not capture the full prevalence pool, because incidence is used as a proxy. Patients being re-treated, or receiving rituximab as second-line treatment, might not be captured-a situation that is less common in DLBCL than in FL. Further, we were unable to use Canadian estimates for time differences in IV compared with sc rituximab administration and patient chair time. However, the U.K. estimates that were used aligned with estimates from a time-and-motion study conducted in 8 countries $^{13}$. In addition, the market-share uptake values for implementation of the sc formulation were based on data estimates, and as seen in the sa, the model is most sensitive to those values. However, despite that sensitivity, we still expect that implementation of the sc formulation will lead to time and cost savings ranging between 114,530 and 216,778 hours in systemic therapy suite time and between approximately $\$ 36$ million and \$68 million dollars over 3 years. Lastly, we did not evaluate the potential additional cost savings associated with the use of sc instead of IV rituximab for other subtypes of CD20-positive NHL.

The strengths of our study include validation of its parameters and assumptions by TAEs and, as much as possible, the use of Canadian-based chart review and literature data in forming estimates.

\section{CONCLUSIONS}

Rituximab in a sc formulation for Canadian patients with DLBCL and FL can reduce the overall burden on systemic therapy suites, making that valuable resource available for the treatment of other patients, while maintaining the safety and effectiveness of rituximab treatment and providing substantial savings in administration costs to the health care system.

\section{ACKNOWLEDGMENTS}

Shainur Premji MSc of the University of Calgary and Marg Hux MSc of Medlior Health Outcomes Research Ltd. provided economic consulting, modelling, and writing. Dominika Dabrowski Msc of DKDA Consulting Inc. conducted the expert interviews. Tara Cowling MSc of Medlior Health Outcomes Research Ltd. provided writing and editorial assistance.

Funding for the present study was provided by Hoffmann-La Roche. Two authors (BM and DB) are Roche employees and were involved in the collection, analysis, and interpretation of the data; in the writing of the report; and in the decision to submit the article for publication collectively as authors.

\section{CONFLICT OF INTEREST DISCLOSURES}

We have read and understood Current Oncology's policy on disclosing conflicts of interest, and we declare the following interests: 
DAS has served on ad hoc advisory boards for Roche, Janssen, Gilead, AbbVie, Celgene, Amgen, Lundbeck, Merck, Bristol-Myers Squibb, Seattle Genetics, and Servier; JSB has served on advisory boards for Janssen, Celgene, Amgen, Roche, and Takeda; BM and DB are Roche employees; RF has participated on ad hoc advisory boards for Celgene, Janssen, Novartis, and Gilead.

\section{AUTHOR AFFILIATIONS}

*Tom Baker Cancer Centre and University of Calgary, Calgary, AB; 'Hôpital du Sacré-Coeur de Montréal, Montreal, QC; ${ }^{\ddagger}$ Hoffmann-La Roche Limited, Mississauga, ON; ${ }^{\S} J u r a v i n s k i$ Hospital and Cancer Centre, Hamilton, ON.

\section{REFERENCES}

1. Canadian Cancer Society. Canadian Cancer Statistics 2017. Toronto, ON: Canadian Cancer Society; 2017.

2. Canadian Cancer Society. Non-Hodgkin Lymphoma Statistics [Web page]. Toronto, ON: Canadian Cancer Society; 2017. [Available at: http://www.cancer.ca/en/cancer-information/ cancer-type/non-hodgkin-lymphoma/statistics/?region=on; cited 17 January 2018]

3. Seiler TM, Hiddemann W. Advances in the management of follicular lymphoma. Curr Opin Oncol 2012;24:742-7.

4. Davies A, Merli F, Mihaljević B, et al. Efficacy and safety of subcutaneous rituximab versus intravenous rituximab for first-line treatment of follicular lymphoma (SABRINA): a randomised, open-label, phase 3 trial. Lancet Haematol 2017;4:e272-82.

5. Molina A. A decade of rituximab: improving survival outcomes in non-Hodgkin's lymphoma. Annu Rev Med 2008;59:237-50.

6. Hiddemann W, Kneba M, Dreyling M, et al. Frontline therapy with rituximab added to the combination of cyclophosphamide, doxorubicin, vincristine, and prednisone (сHOP) significantly improves the outcome for patients with advanced-stage follicular lymphoma compared with therapy with cHор alone: results of a prospective randomized study of the German LowGrade Lymphoma Study Group. Blood 2005;106:3725-32.

7. Pfreundschuh M, Trümper L, Österborg A, et al. on behalf of the MabThera International Trial Group. CHOp-like chemotherapy plus rituximab versus CHOP-like chemotherapy alone in young patients with good-prognosis diffuse large-B-cell lymphoma: a randomised controlled trial by the MabThera International Trial (MInT) group. Lancet Oncol 2006;7:379-91.

8. Davies A, Merli F, Mihaljevic B, et al. Pharmacokinetics and safety of subcutaneous rituximab in follicular lymphoma
(SABRINA): stage 1 analysis of a randomised phase 3 study. Lancet Oncol 2014;15:343-52.

9. Rule S, Collins GP, Samanta K. Subcutaneous vs intravenous rituximab in patients with non-Hodgkin lymphoma: a time and motion study in the United Kingdom. J Med Econ 2014;17:459-68.

10. Macdonald D, Crosbie T, Christofides A, AssailyW, Wiernikowski J.ACanadian perspective on the subcutaneous administration of rituximab in non-Hodgkin lymphoma. CurrOncol2017;24:33-9.

11. Hoffmann-La Roche Ltd. Rituxan SC [product monograph]. Mississauga, ON: Hoffmann-La Roche; 2016.

12. Salar A, Avivi I, Bittner B, et al. Comparison of subcutaneous versus intravenous administration of rituximab as maintenance treatment for follicular lymphoma: results from a two-stage, phase Iв study. J Clin Oncol 2014;32:1782-91.

13. De Cock E, Kritikou P, Sandoval M, et al. Time savings with rituximab subcutaneous injection versus rituximab intravenous infusion: a time and motion study in eight countries. PloS One 2016;11:e0157957.

14. Institut national d'excellence en santé et en services sociaux Québec (INESSS). Rituxan SC - Lymphome non hodgkinien $(L N H)$ [ministerial notice, French]. Quebec City, QC: INEsss; 2017. [Available online at: http://www.inesss.qc.ca/fileadmin/ doc/INESSS/Inscription_medicaments/Avis_au_ministre/ Octobre_2017/RituxanSC_2017_10.pdf; cited 15 August 2018]

15. Rummel M, Kim TM, Aversa F, et al. Preference for subcutaneous or intravenous administration of rituximab among patients with untreated CD20+ diffuse large B-cell lymphoma or follicular lymphoma: results from a prospective, randomized, open-label, crossover study (PrefMab). Ann Oncol 2017;28:836-42.

16. Statistics Canada. Estimates of population, by age group and sex for July 1, Canada, provinces and territories, annual (persons unless otherwise noted). CANsim table 051-0001. Ottawa, ON: Statistics Canada; 2017.

17. Tam VC, Ko YJ, Mittmann N, et al. Cost-effectiveness of systemic therapies for metastatic pancreatic cancer. Curr Oncol 2013;20:e90-106.

18. Ontario Ministry of Health and Long Term Care (монцтC). Schedule of Benefits: Physician Services under the Health Insurance Act. Toronto, ON: MOHLTC; 2015.

19. Lugtenburg P, Avivi I, Berenschot H, et al. Efficacy and safety of subcutaneous and intravenous rituximab plus cyclophosphamide, doxorubicin, vincristine, and prednisone in firstline diffuse large B-cell lymphoma: the randomized MabEase study. Haematologica 2017;102:1913-22. 